\title{
Interplay Between Deposition, Overhang, Evaporation and Surface Diffusion in A Growth Model for two Species in (2+1)-Dimensions
}

\author{
Hassan F. El-Nashar \\ Department of Physics, Faculty of Science, Ain Shams University, 11566 \\ Cairo, Egypt
}

\begin{abstract}
We propose a surface growth model for binary species in $(2+1)$ dimensions, in which we consider the effect of the interplay between deposition, overhang, evaporation and surface diffusion processes on the kinetics of growth. Numerical simulations show that the extracted exponents deviate from the known universality classes. The model shows that when evaporation occurs frequently, the surface tends to be rougher even in the presence of surface relaxation.
\end{abstract}




\section{Introduction:}

The growth of surfaces and interfaces remains a challenging problem in physics. It attracts interest due to its technological importance because many properties of real materials depend on the presence of surface and interface roughness. During the growth, the surface of materials becomes undesirably rough. In order to avoid this morphology, the basic physical effects and the processes that lead to the development of surface roughness must be well understood. Apart from specific technological applications, most rough surfaces are formed under conditions that are far from equilibrium. Therefore, the study of roughening has a relevant importance in the understanding of nonequilibrium statistical mechanics at the fundamental level $[1,2]$.

Simple discrete growth models have played a major role in the understanding of the nature of surface roughness since they mimic the essential physical parameters and eliminate details which are less important. Although surface growth models have been extensively studied in the past, most of previous studies have dealt with the deposition of only one component. The growth of two or more species is common in modern technology where little is known about the kinetic of roughening $[3,4]$. Therefore, the study of such problems is important since it gives a new insight into the dynamics of roughening. In this case the surface growth kinetics reflects how the surface roughens during its evolution with time. Most studies of composite growth have been carried out in $(1+1)$-dimensions. In order to be closer to the real systems, one needs to perform simulations in $(2+1)$-dimensions. Another important fact is that there are still disagreements about the classes of the universality to which certain models belong and we may expect to find a deviation from the known universality classes [3,4]. Even though the studies regarding the growth of alloys were designed to include processes such as deposition and surface relaxations, there could be evaporation processes in the real growth, as well as chemical vapor deposition. The presence of evaporation may affect strongly the surface roughening. Models describing such phenomenon are rarely studied in the case of two components even if this evaporation process is as important as deposition and surface relaxations [5,6]. Previous numerical studies, that take evaporation process into consideration, were only performed in $(1+1)$ dimensions using solid-on-solid models where overhangs are not allowed $[5,6]$, although it is a possible process, especially at a high angle of incidence [7]. Moreover, in (2+1)-dimensions, overhang/voids are entropically favored [8].

It is well known that a stochastically growing surface exhibits scaling behaviour and evolves to a steady state without a characteristic time or length scale. Starting from an initially flat substrate and defining the surface width as 


$$
W^{2}(L, t)=\frac{1}{L^{d-1}} \sum_{r}[h(r, t)-\overline{h(t)}]^{2}
$$

where $L$ is the substrate size, $h(r, t)$ is the surface height at position $r$ and time $t$, $\overline{h(t)}$ is the average height at time $t$ and $(d-1)$ is the substrate dimension, the scaling law [1] is given by $W(L, t)=L^{\alpha} f\left(t / L^{z}\right)$. The exponents $\alpha$ and $z$ are the roughness and dynamic exponents, respectively. The scaling function $f(x)=x^{\beta}$ for $x<<1$, where the growth exponent is defined as $\beta=\alpha / z$, and $f(x)=$ const. for $x>>1$. This scaling behaviour has been studied in various systems and models and has been argued to be universal [2]. Additionally, the extracted values of exponents reflect a direct information about the surface morphology.

Several studies for the growth of binary mixtures have been made in the context of the ballistic deposition model (BD) where overhang processes are relevant. Here, we propose to study the effect of evaporation processes in the presence of overhang and surface relaxation on surface roughening in $(2+1)$ dimension. We focus on the interplay between these processes and how the interaction between the two different particles affects the kinetics of growth. We introduced the above mentioned processes in a simple model for two species without including any other details, which might be found in real systems, in order to see clearly how the surface evolves with time according simply to the competition between the three main processes. It is known that the dynamics of both species is important as well as the processes such as deposition, evaporation, overhang and incorporation, etc., which increases the level of difficulty of the problem, to consider all of them at once. Therefore, the use of a rather simple model for two species which includes only simple processes, will give insight into the dynamics of roughening only in the presence of the processes under investigations. We believe that the evaporation process in the case of two species of different interactions in addition to overhang/void and surface diffusion will affect the surface morphology. Henceforward, one can get answers to the following questions: "does the interplay between deposition, overhang, evaporation and surface diffusion affect the kinetic roughening? and is evaporation an important process to be considered in the case of surface growth of two species?".

The organization of this paper is as follows: in section II we describe the model and specify the growth of the two species according to the processes of deposition, evaporation and overhang. In section III we present the results of simulations. Finally, in section IV the conclusion are given. 


\section{The Model:}

We propose a model adequate to describe reactions which take place on the growing surface of materials. It represents the surface growth due to the deposition of two kinds of particles with different attractive forces where the temperature is sufficiently high such that the less active particle is allowed to evaporate. The model does not describe the growth of a specific material, it concentrates on the interplay between basic processes during the growth. In order to model the growth of a real material, e.g., semiconductors [9], some details should be included which will make the model complicated, thus making difficult to analyse the effect of basic processes under considerations.

In general, the rules of interaction are such that one type of particles (A) is always necessary for the deposition of both species to occur, while the other (B) is allowed to evaporate or to control the diffusion across the surface. We introduce two versions of the model: model (I), simply allows depositionevaporation and overhang, while model (II) allows surface relaxation after deposition of one type of species onto another. The growth process is as follows: at first a column is selected at random and then a particle (A) (or particle (B)) is deposited on the surface with a probability $(1-P)$ (or $(P)$ ); i.e, the deposition rate of each particle depends on the chosen probability $(P)$. A cross section of the aggregate is shown in Fig. (1). The white squares represent the aggregated particles of type (A) and dark squares represent those of type (B). Dashed squares represent the places of deposition of the newly arrived particles. Circles denote the incoming particles while the down arrows represent deposition. Evaporation is indicated by the inclined dotted arrows. According to model (I) the aggregation occurs as shown in Fig. (1a): a particle of type (A) will stick to the first particle (A) or (B) that meet, either at the top of the chosen column (particles 1,2,5 and 6) or sideways (particles 3, 4 and 7). When the incoming particle is of type (B), it sticks to the top of the chosen column if the latter contains particle (A) (particles 5 and 6) or sticks laterally to one of the neighboring sites higher by one step if that contains a particle of type (A) (particle 8). Otherwise, it is evaporated if it finds only a particle (B) (particle 1 and 2). When the chosen column is lower than its neighbors, (B) will stick laterally if it finds a particle (A) in the neighboring columns (particles 3, 7 and 8) or it evaporates (particle 4) if there is no particle (A) along any of the surrounding neighboring sites. For model (II), the rules of deposition, evaporation and surface relaxation, depicted in Fig. (1b), are the same as the rules presented for model (I) except that surface relaxation is considered when there exists lower spaces available. In these situations the incoming particle (A) or (B) remains on top or sticks laterally to a particle of another type, then this incoming particle relaxes to a minimal position (particles 2,6 and 7) or downwards (particle 4 and 7 ). 


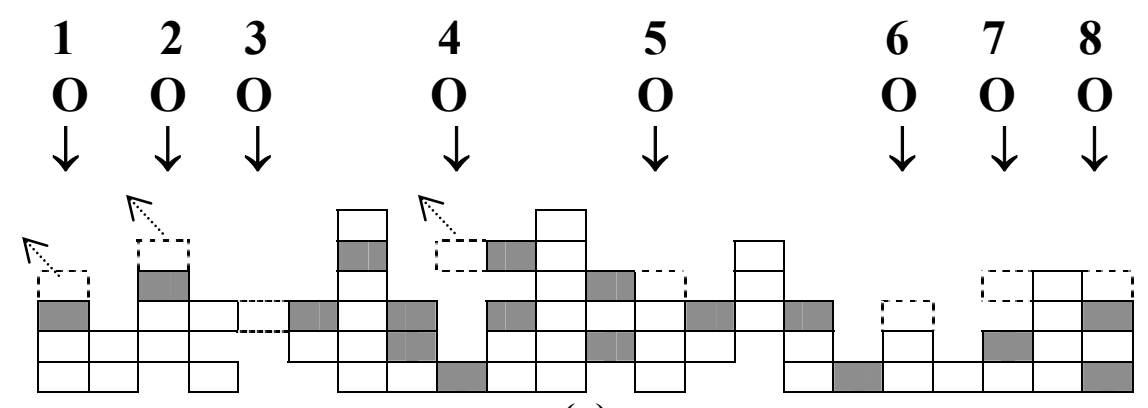

(a)

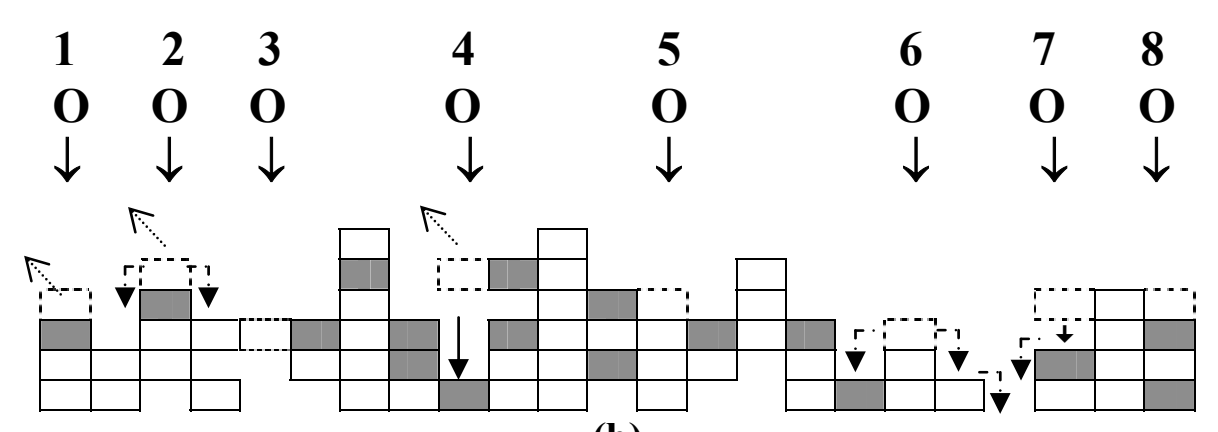

(b)

Fig. (1): (a) A piece of the cross section of the aggregate according to model I (b) A piece of the cross section of the aggregate according to model II.

\section{Results:}

The simulation is performed on a square lattice $L \times L$ on the $X Y$-plane with periodic boundary conditions such that $h(X+i L, Y+j L)=h(X, Y)$, where $h(X, Y)$ describes the height function of the surface, for any integer values of $i, j$. The aggregation occurs in the $Z$ direction. Statistical average is obtained over 300 independent simulations for each parameter. The simulation is carried out on the computer by means of a Monte-Carlo method. In order to simulate the processes according to models (i) and (ii), a computer program is written using the FORTRAN language. We use different random number generators from CERN and EMSSL libraries as well as a code written by the author in order to satisfy the following conditions: a) the random numbers are uniformly distributed in the interval [0-1] and b) these random numbers are uncorrelated. The computer program has been tested and executed on several platforms such as Sun Solaris, IBM/R6000 and ALPHA Digital machines. Throughout the code of the computer program, a three-dimensions array is used to represent the embedded dimensions $d$. The first two dimensions of the array represent the 
substrate of dimensions $d-1$ and the third dimension is used for the height of the aggregation. The site on the substrate of location $(i, j)$ is chosen randomly according to a specific model and then it is filled by a particle of type (A) or (B) according to the value of $P$ or $(1-P)$. The deposition, diffusion or evaporation processes are determined according to the content of the surrounding sites of the newly filled particle in the location $(i, j)$. The time is computed as one MonteCarlo step. At each Monte-Carlo step, the height $h(r, t)$ at each site and the average height $\overline{h(t)}$ of all aggregation are calculated in order to get finally the value of the surface width $W(L, t)$ at this time.

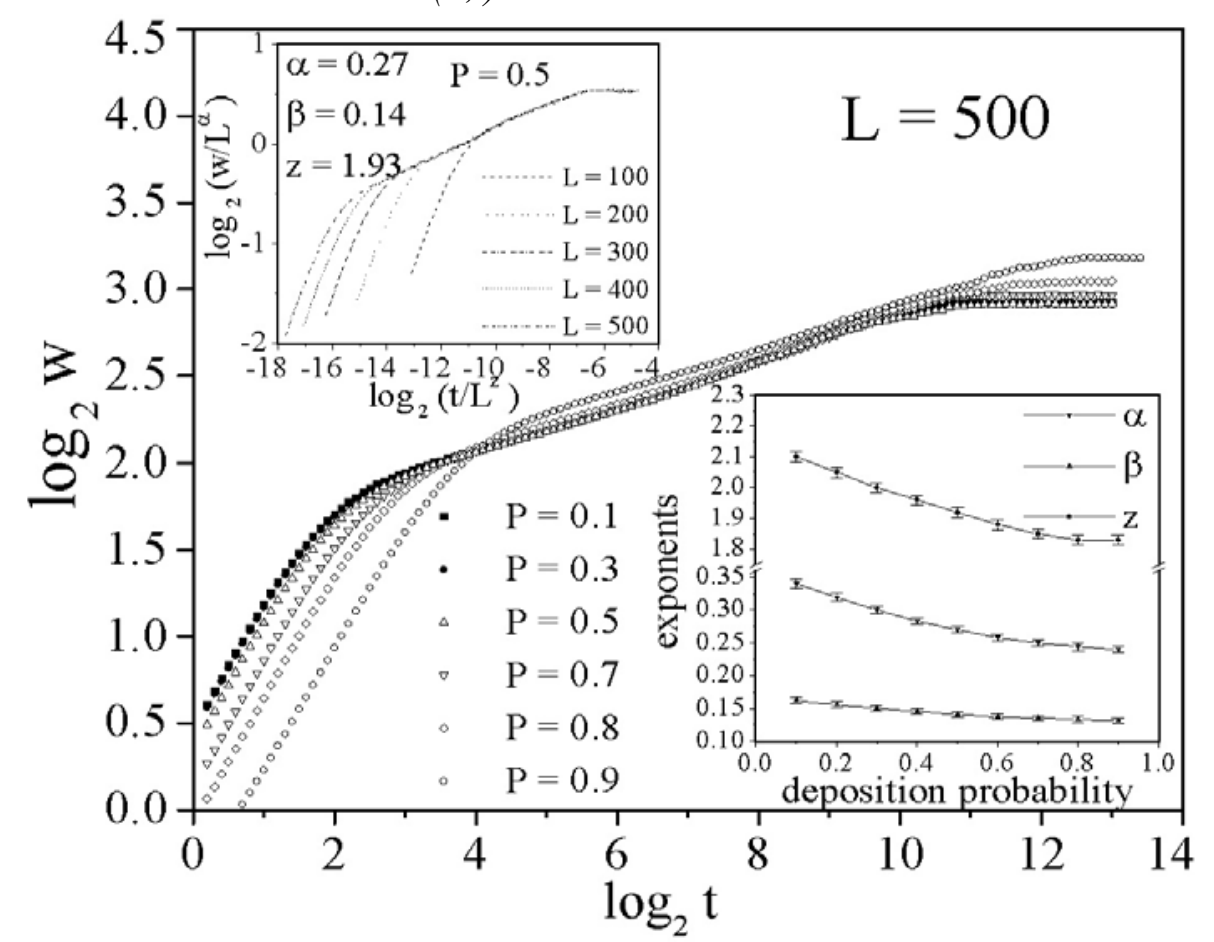

Fig. (2): $L o g-\log$ plot of the surface width versus time for different $(P)$ for $L=500$ for model I. The upper inset shows the collapse of all plots when $W$ and $L$ scaled according to the extracted exponents for $P=0.5$. The lower inset shows the values of the exponents versus the deposition probability.

The results for model (I) are shown in Fig. (2): a log-log plot of the surface width $W$ as a function of time (number of deposited layers) for different values of the deposition probability $(P)$ and fixed substrate side size $L_{X}=L_{Y}=500$. The surface width appears to be fixed until $P=0.5$ and then it increases but not to a very high value. We also notice that $W(t)$ shifts to the right for $P>0.5$ for short times. The upper inset of Fig. (2) shows the values of the 
exponents $\alpha, \beta$ and $z$ determined for $P=0.5$ while in the lower inset the values of the exponents are plotted versus the deposition probability $(P)$. Fig. (3) shows the log-log plot of the surface width versus time for model (II) for different values of $(P)$. It is seen that, at long times, the surface width increases when $(P)$ increases while it shifts towards the right for shorter times. As it appears from both figures, the growth stages are divided into three regimes. We consider the first stage as transient [2]. Fig. (4) represents the saturated surface width $W_{s}=W(t=\infty)$ when it is plotted versus the deposition probability $(P)$ for both models. It is seen from Fig. (4) that for model (I), $W_{S}$ remains approximately unchanged for $P<0.5$ and then it increases. For model (II), $W_{S}$ is a monotonically increasing function of $(P)$.

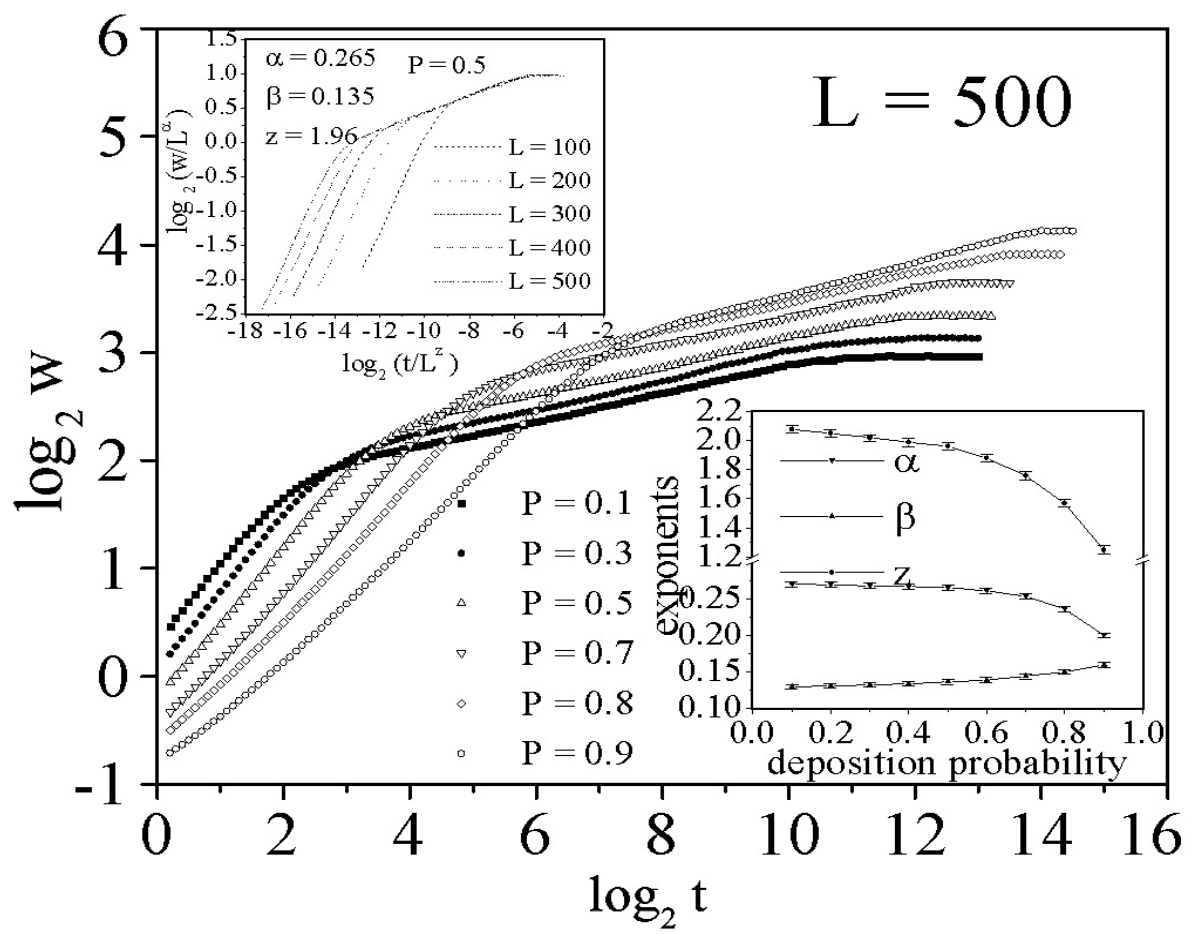

Fig. (3): Log-log plot of the surface width versus time for different $(\mathrm{P})$ for $L=500$ for model II. The upper inset shows the collapse of all plots when $W$ and $L$ scaled according to the extracted exponents for $P=0.5$. The lower inset shows the values of the exponents versus the deposition probability.

A successful approach to describe the surface evolution in the case of ballistic deposition model for one kind of deposited particles is the KardarParisi-Zhang (KPZ) [10] equation, 


$$
\frac{\partial h}{\partial t}=v \nabla^{2} h+\frac{\lambda}{2}(\nabla h)^{2}+\eta(r, t)
$$

which is composed of a linear term $\nabla^{2} h$ and a nonlinear term $(\nabla h)^{2}$ plus a stochastic term which represents the randomness of the deposition processes. The (KPZ) equation gives what is so called the (KPZ) universality with exponents $\alpha \approx 0.37$ and $\beta \approx 0.24$. The values of the exponents for models (I) and (II) show deviations from the (KPZ) universality, for any value of $(P)$. For model (I), the main processes responsible for the growth are deposition, overhang and evaporation. For the second model there is an extra process on the surface which is surface diffusion. It is clear from the values of the $\beta$ exponents for both models that the kinetics of the growth becomes slower than that of (BD) model of one kind of species. The exponents $\alpha$ indicate that the surface features are also different.
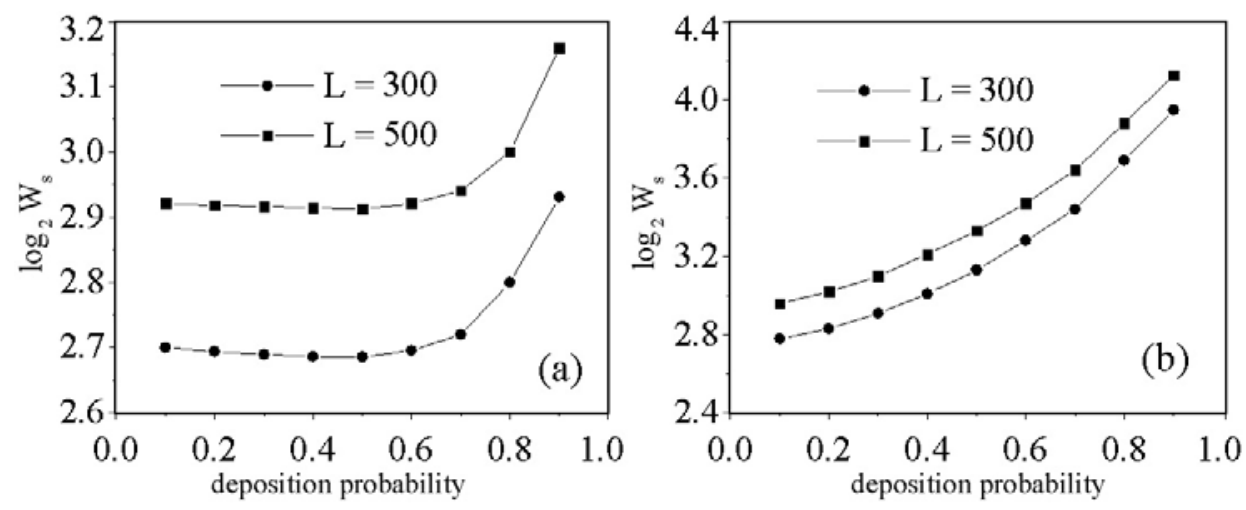

Fig. (4): $L$ Log of the saturated surface width versus deposition probability $(P)$ for $L=300$ and $L=500$ (a) model (I) and (b) model (II).

Clearly, overhang increases the local slopes on the surface which in turn enhance the nonlinear term in the (KPZ) equation. However, the surface relaxation and evaporation strengthen the linear term [2]. Therefore, the deviations in the exponents from those determined by KPZ maybe due to the competition between the different processes. In order to get more information about the kinetic behaviour in both models, we calculated the average velocity of the interface for different values of $(P)$, which is presented in Fig. $(5 \& 6)$ in a log-log plot versus $t$ for models (I) and (II), respectively. It is clear from both figures that the average interface velocity is reduced as $(P)$ increases (it decreases even more in the presence of diffusion) but it does not become zero and it is less than that of (BD) for one species [4]. It can also be seen from 
Fig. $(5 \& 6)$ that the interface advances more slowly in the presence of surface relaxation. Therefore, it takes longer to the interface velocity for the model with surface diffusion (II) to reach a constant value than to that of model (I) (see Figs. 2 and 3). This may give some insight to understand why $\beta$ is smaller in model (I), and larger in model (II), when evaporation becomes relevant, i.e, for larger value of $(P)$. The surface width grows in the intermediate stage (see Figs. 2 and 3 ) as $W \cong t^{\beta}$. According to Fig. (5\&6), the surface width grows faster in the case of model (I) than in that of model (II). However, during the intermediate stage it takes a longer time for model (I) to reach a relatively lower steady state in comparison to that of model (II). In the case of model (II), the interface proceeds with lower velocity, and its surface width reaches to a relatively higher value of a steady state in a relatively shorter time (compare Figs. 2 and 3). Therefore, the exponent $\beta$ in the first model is lower than that of model (II) for the same parameters when evaporation becomes dominant. The higher value of the saturated surface width for model (II) relative to that of model (I) indicates that the surface in the case of the model with diffusion is more jagged than that of the case without diffusion.

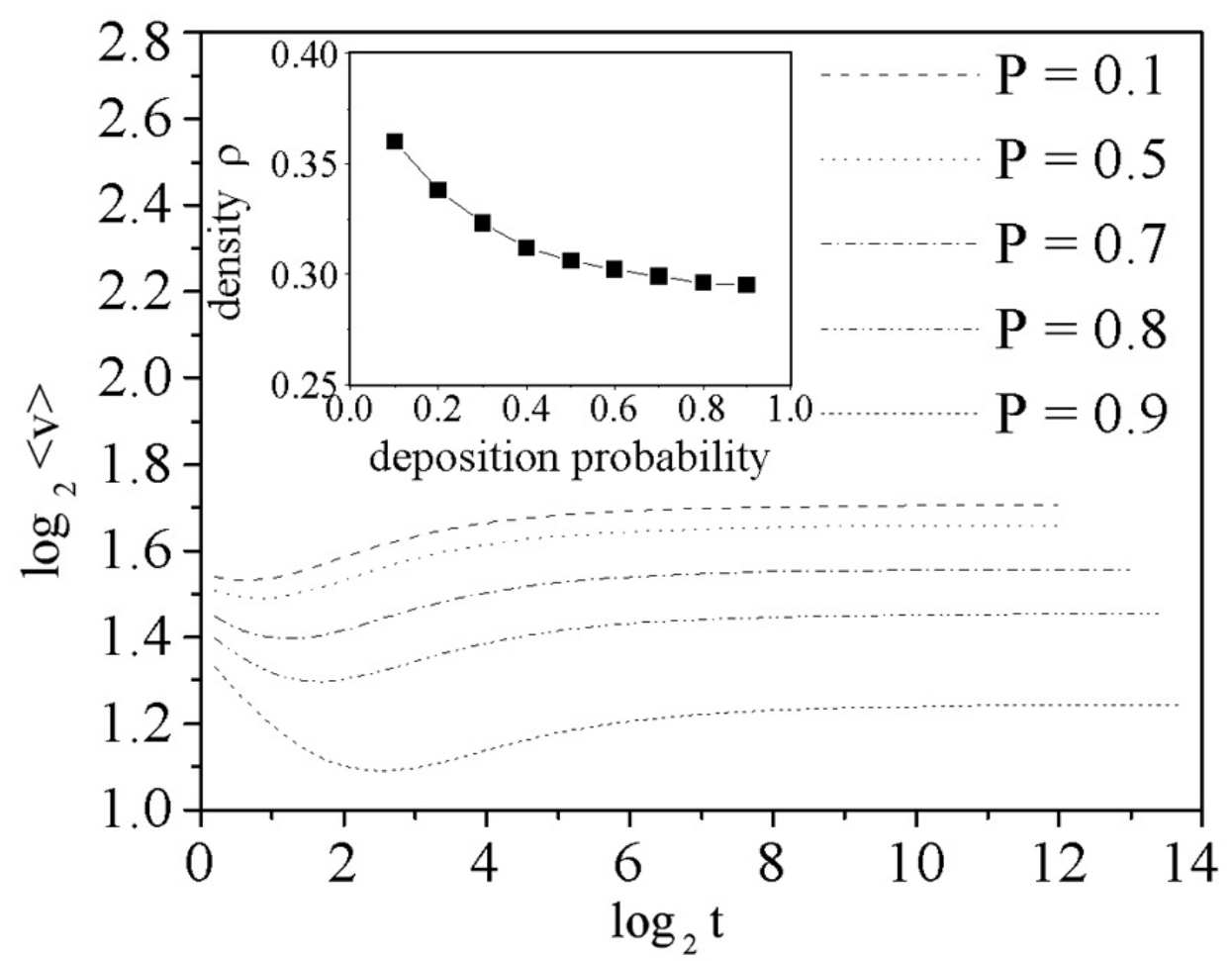

Fig. (5): Log-log plot of the average interface velocity versus time for model I for different $(P)$. The inset shows the density $\rho$ versus the deposition probability $(P)$. 


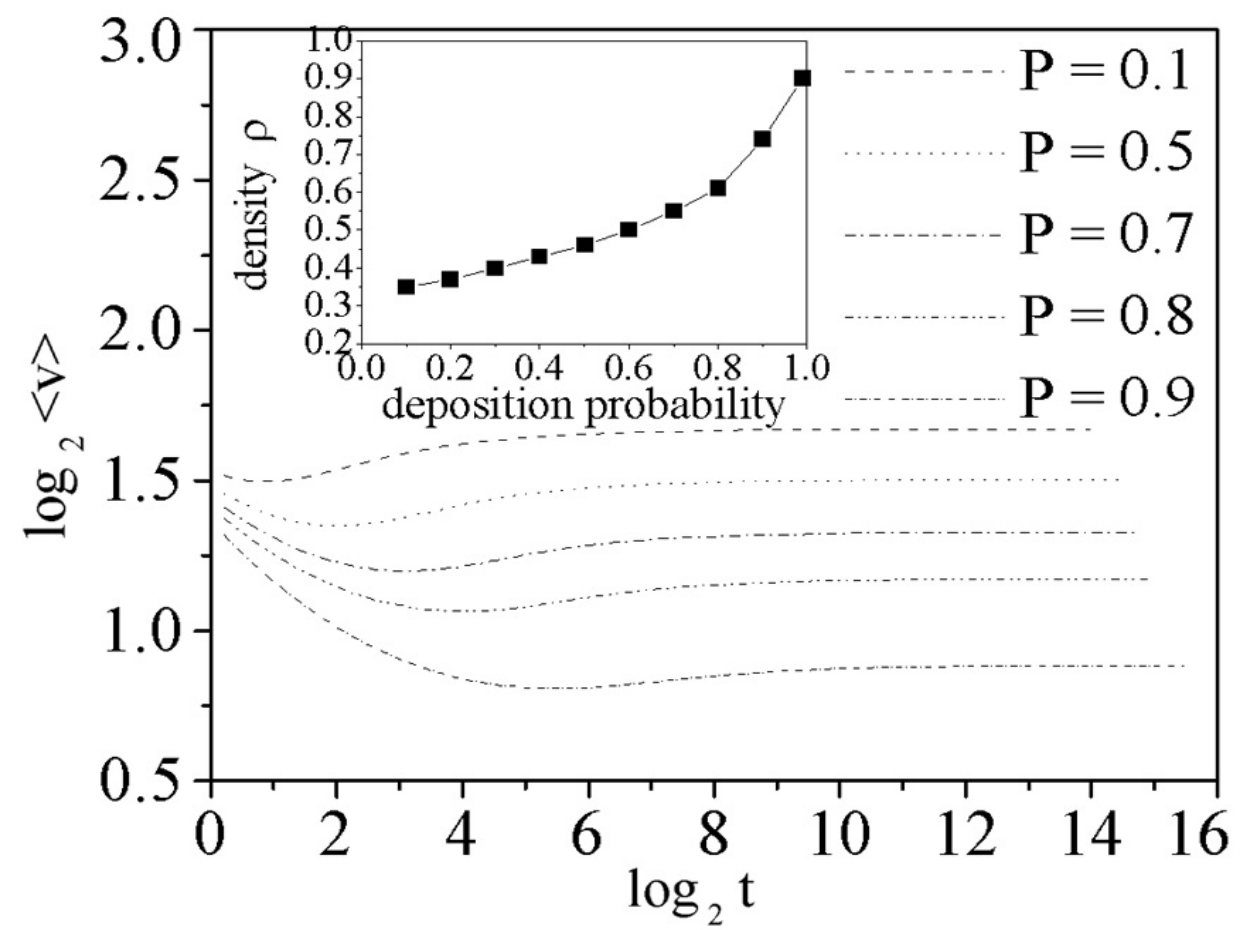

Fig. (6): Log-log plot of the average interface velocity versus time for model II for different $(P)$. The inset shows the density $\rho$ versus the deposition probability $(P)$.

It is known that when voids/overhang processes become relevant, the surface width grows faster [2] since it increases the nonlinear term $(\nabla h)^{2}$ in the (KPZ) equation, which enhances the excess velocity. In order to get more information about such processes, we investigated the density $\rho=N / \bar{h} L^{2}$ of the whole aggregate in both models for different values of $(P)$ (see insets of Figs. 5 and 6). It is seen from figure 5 that the density in the first model decreases slightly with the probability while the change in the velocity is more dramatic. We argue that the evaporation process, when it happens frequently, affects the growth kinetic more than the overhang/voids, that the interface advances depend on the presence of particles (A) (rarely deposited) and that evaporation slows down the motion of the interface. This happens since overhang enhances the local slopes on the surface while evaporation reduces these local slopes and increases the linear term $\nabla^{2} h$. On the other hand, the density increases as $(P)$ increases as shown in figure 6 . We conclude that it increases due to voids filling by diffusion under the surface. The excess velocity, which is not equal to zero, indicates the presence of nonlinearity in the growth. In this case evaporation and diffusion are responsible for the reduction in the interface velocity. Notice that 
surface diffusion occurs more frequently to particles (A), since (B) are allowed to evaporate. Thus, columns that contain particles of type (A) grow more than those containing (B). Therefore, surface diffusion enhances the height fluctuations to be higher over the surface. This gives another reason for $\alpha$ to be smaller and for the surface to be rougher in the second model as well as for $\beta$ to have a higher value.

\section{Conclusion:}

In conclusion, we have shown through the study of different parameters that when evaporation is considered in the surface growth, in addition to overhang and diffusion, the exponents clearly deviate from that of the (KPZ) universality class. Through the study of different quantities, surface width, average velocity, density, we have explained how each process affects the surface roughening leading to a different kinetics. Qualitatively, the deviation from the known universality may relate to the different rules of interactions between different species [6]. We have done simulations for larger system size, $L=1000$ and have not found any sign of a crossover from our results into KPZ universality. We noticed that, the presence of evaporation and relaxation on the surface reduces the local gradient on the surface. This local gradient if it is only due to overhang, will enhance the growth kinetics and lead to a faster growth. However in model (I), the presence of evaporation reduces the effect of the overhang and slows down the kinetics. In the case of model (II) it was expected that the evaporation and surface diffusion will lead to an even slower growth kinetic. However, the surface width increases even more than that of model (I), due to the inhomogeneities produced by the distribution of the different species on the surface.

\section{Acknowledgement:}

HFE would like to thank the MPIPKS, Dresden, Germany for support during some part of this work.

\section{References:}

1. F. Family, T. Vicsek, "Dynamics of Fractal Surfaces", World Scientific, Singapore, (1991).

2. A. L. Barabasi and H. E. Stanley, "Fractal Concepts in Surface Growth", Cambridge University Press, Cambridge, (1995).

3. H. F. El-Nashar, W. Wang and H. A. Cerdeira, Phys. Rev. E 58, 4461 (1998).

4. H. F. El-Nashar and H. A. Cerdeira, Phys. Rev. E, 60, 1262 (1999).

5. S. H. Yook and Y. Kim, Phys. Rev. E, 60, 3837 (1999). 
6. S. Park and B. Kahng, Phys. Rev. E, 60, 6160 (1999).

7. S. W. Levine, J. R. Engstrom and P. Clancy, Surf. Sci., 401, 112 (1998).

8. M. Marsili and Y. C. Zhang, Phys. Rev. E, 57, 4814 (1998).

9. P. Politi, G. Grenet, A. Marthy, A. Ponchet and J. Villain, Phys. Rep., 324, $271(2000)$.

10. M. Kardar, G. Parisi and Y. C. Zhang, Phys. Rev. Lett., 56, 889 (1986). 